\title{
Synthesis of Boron Nitride Nanotubes by arc-jet plasma
}

\author{
C. M. Lee*, S. I. Choi, S. S. Choi, and S. H. Hong** \\ Department of Nuclear Engineering, Seoul National University \\ Seoul 151-742, Korea
}

\begin{abstract}
Boron nitride nanotubes (BNNTs) are synthesized by the arc-jet plasma of high temperature $(5000-20000 \mathrm{~K})$, which is generated by a dc non-transferred plasma torch with a mixture of argon and nitrogen gas. The BNNTs produced by the thermal plasma process are resulted from the chemical reaction of a precursor material of hexagonal boron nitride with catalytic metal powders of $\mathrm{Ni}$ and $\mathrm{Y}$ introduced into the hot arc-jet plasma flame, which is ejected inside the cylindrical carbon reactor. The synthesized materials are evaluated by scanning electron microscopy (SEM), transmission electron microscopy (TEM), and electron energy loss spectroscopy (EELS). These material analyses reveal that multi-walled nanobubes including single- and double-walled ons with high crystallinity are synthesized in quantity, and that the diameter of nanotubes is estimated in the range of $3-10 \mathrm{~nm}$.
\end{abstract}

PACS : $52.75 \mathrm{Rx}, 81.05 \mathrm{Ys}$

E-mail :*maver1@snu.ac.kr, **hongsh@snu.ac.kr

Fax : *+82-2-877-1343

Keywords: boron nitride nanotubes, arc-jet plasma, non-transferred plasma torch 


\section{Introduction}

Based on the structural similarity between carbon and hexagonal boron nitride, the research on the synthesis of boron nitride nanotubes (BNNTs) has attracted great attention to the development of its efficient, large-scale processes since the first discovery of carbon nanotubes [1]. BNNTs have a mechanical strength of $1.2 \mathrm{TPa}$ [2] similar to that of carbon nanotubes (CNTs), superior thermal stability and oxidation resistance. In addition, BNNTs exhibit a stable band gap of $\sim 5.5 \mathrm{eV}$ independent of the tube diameter, chirality, and the number of layers [3], whereas CNTs are metallic or semiconducting depending on their morphology. Therefore BNNTs have a great potential of different technological applications to high performance electronic devices, insulating nanotubular shield [4], hydrogen storage medium [5], and so on.

BNNTs have been usually synthesized using the methods similar to those used for forming CNTs, such as arc discharge [6], laser ablation [7], and chemical vapor deposition (CVD) [8]. Recently, many other synthesis methods have also been proposed such as substitution of CNTs [9], template confined methods [10], and mechano-thermal methods [11]. However, it is likely that there is no reliable method to produce large amounts of BNNTs with high purity and quality. The synthesis processes by CVD or related chemical methods are relatively simple and possible at low temperature range $(<$ $1700 \mathrm{~K}$ ), but produce the synthesized materials having bamboo-like structure, poorly crystalline or polycrystalline walls, and large diameter above $50 \mathrm{~nm}$ [12]. The arc discharge method can produce highly crystallized nanotubes with reduced number of layers and small diameter in the high temperature ambience (above $5000 \mathrm{~K}$ ) between two consumable electrodes composed of BNNTs precursor materials [13]. However, because of electrically insulating property of boron or most boron compounds, the electrodes as a precursor have been limited to metallic borides [6] or boron nitride packed into a metal casing [14] or specially prepared electrode containing boron [13]. In addition, the electrodes as precursor are consumed by arc erosion, and the processing time is limited to the lifetime of electrodes. Therefore, these methods necessarily result in serious drawbacks of poor purity, overabundance of metal catalyst, and noncontinuous process.

In this paper, we present a new promising technique for continuous large-scale production of BNNTs by the arc-jet plasma process using a non-transferred plasma torch [15]. The arc-jet thermal plasma is generated by introducing a cold plasma 
forming gas into the current path of dc arc discharge between two metal electrodes. In our new method of arc-jet plasma, the metal electrodes are not used as boron precursor to supply a vapor-phase boron source, and the thermal plasma jet is used just as a heat source. The lifetimes of the tungsten cathode and copper nozzle anode used in the torch are very long compared with those of the consumable electrodes used in the conventional arc method. And the boron and catalyst sources are introduced from the injectors into the thermal plasma jet independently of the arc discharge itself. So the feedstock can be continuously supplied for the BNNTs synthesis. In addition, the thermal plasma characteristics, such as temperature, enthalpy and velocity, of the arc jet flame can be more easily controlled and diagnosed than the arc characteristics of conventional arc-discharge methods. Therefore, this new method is more favorable to the research on the BNNTs formation mechanism. Shimizu et al. also have proposed a similar method to our arc-jet thermal plasma method, but they have used a bulk boron compound target, which makes it impossible for their method to be operated in a continuous process [16].

In this paper, we present a successful synthesis of BNNTs by the thermal plasma process with a mixture powder of boron nitride precursor, and $\mathrm{Ni}$ and $\mathrm{Y}$ metal catalysts injected into the hot arc-jet plasma flame in the cylindrical carbon reactor. The formation and morphology of the synthesized BNNTs have been evaluated by scanning electron microscopy (SEM), transmission electron microscopy (TEM), and electron energy loss spectroscopy (EELS).

\section{Experimental}

A schematic diagram of BNNTs synthesis system is depicted in Fig. 1. The experiment system mainly consists of a dc non-transferred plasma torch, injector of reactant materials and catalysts, and reaction cylinders made of carbon. The arc-jet plasma is generated by arc discharge between a tungsten cathode of conical shape and a copper anode of cylindrical nozzle shape in the dc plasma torch, and the resultant arc-jet thermal plasma is ejected from the anode nozzle exit into the inside of the reaction cylinders jointed together in front of the anode nozzle exit. Both electrodes are cooled by water in order to prevent them from erosion caused by enormous heat flux from the arc. A mixture gas of argon and nitrogen is used as a plasma forming gas. Argon and nitrogen flow rates are $45 \mathrm{slpm}$ and $2 \mathrm{slpm}$, respectively. The reactant materials are prepared as a mixture of hexagonal boron nitride powder (99\%, $1 \mu \mathrm{m}$, Aldrich) as 
boron source material and $\mathrm{Ni} / \mathrm{Y}$ powder (atomic ratio 9:1) as catalytic materials. The reactant mixture powder is introduced by argon carrier gas into the arc-jet plasma flame through the injection holes at a reaction cylinder near the nozzle exit, and undergoes synthesis reactions in the high temperature plasma flowing through the inside of the reaction cylinders. The reaction cylinders made of carbon are installed to build up a long reaction zone of high temperature, and thus enhance the synthesis reactions among the reactants. The five cylinders with $80-\mathrm{mm}$ length, $12-\mathrm{mm}$ inner diameter, and $25-\mathrm{mm}$ outer diameter are serially connected.

The arc-jet plasma torch is operated with a dc current of $300 \mathrm{~A}$ and an applied voltage of $46 \mathrm{~V}$. The ambient pressure is maintained at atmospheric condition. After the synthesis experiment, the gray products deposited on the inner wall of the reaction cylinders are collected and observed by field emission SEM (JEOL, JSM-6330F), high resolution TEM (JEOL, JEM-2100F, 200 kV), and EELS (Gatan, GIF 2001).

\section{Result and Discussion}

SEM and TEM images of the samples collected from the wall of reaction cylinder II are shown in Figs. 2(a) and (b), which indicate that there is an abundant quantity of entangled nanotubular structures. The nanotubes are not observed in the collected samples in other reaction cylinders. The nanotubes have outer diameters of $3-10 \mathrm{~nm}$ and lengths of up to several $\mu \mathrm{m}$. A bamboo-like structure, of which the inside is divided into several segments by compartment layers, is not observed, and most of nanotubes have encapsulated catalyst particles at their tip.

The composition and structure of the produced nanotubes are verified by elemental mappings and spectrum of with EELS in Fig. 3. Figure 3(a) is the zero-loss energyfiltered TEM image and the corresponding elemental maps of boron and nitrogen are shown in Figs. 3(b) and (c), respectively. In the elemental maps, the differences in brightness at the wall, inside, and outside of the tube apparently reveal that the produced materials have a tubular structure and are composed of boron and nitrogen species. Because the nitrogen map is acquired after boron mapping, a deformation in the nitrogen map is observed due to electron irradiation damage. Figure 3(d) shows a representative EELS spectrum of produced nanotubes. The spectrum displays two distinct K-shell ionization edges for boron and nitrogen starting at $188 \mathrm{eV}$ and $401 \mathrm{eV}$, respectively. Weak peaks of carbon K-edge near $284 \mathrm{eV}$ is likely to come from carbon 
coated to TEM grid. The fine structures of the spectrum are $\pi^{*}$ peaks (peculiar to $1 \mathrm{~s} \rightarrow$ $\pi^{*}$ electron transitions) on the left side of the edges and $\sigma^{*}$ zones (peculiar to $1 \mathrm{~s} \rightarrow$ $\sigma^{*}$ electron transitions) on the right side of the edges, and are the characteristic of $\mathrm{sp}^{2}$ hybridization state [17]. The sharp $\pi^{*}$ peaks of the acquired spectrum confirm the formation of h-BN.

Fig. 4 shows various high resolution TEM images of the nanonubes including single walled (a) and double walled nanotubes (b). The single or double walled nanotubes are well known to be produced by high temperature methods, such as conventional arc discharge methods and laser ablation methods, and hardly observed in the reports using CVD or related chemical methods including a similar arc-jet plasma method [16] with our method. But, the single or double walled nanotubes quite easily observed in our study. Also, the nanotubes show very well-aligned atomic layers along the tube axis and high crystallinity compared with the products using low temperature methods, such as CVD and ball mill methods. The inter-layer spacings in the tube lattice fringes are measured to be $\sim 0.34 \mathrm{~nm}$, which is consistent with the inter-plane distance of (002) in bulk h-BN. The formation of nanotubes having reduced number of layers and high crystallinity seems to be owing to sustaining high temperature condition of arc-jet plasma through the reaction cylinders. Fig, 4 (c) and (d) show the catalyst particles existing in different types. Whereas the particle of Fig. 4 (c) is not encapsulated with $\mathrm{BN}$ layers and the $\mathrm{BN}$ layers is grown from the particle, the particle of Fig. 4 (d) is wholly encapsulated and the growth of BN layers from the particle is unclear. But, both particles show same amorphous morphology, which means that the catalyst particles exist as liquid state during the plasma operation and are not enough crystallized because of rapid cooling over the plasma operation. This indicates that the temperature in the reaction cylinder, in which the nanotubes are grown, is in the range from the melting temperature or eutectic melting temperature of catalysts to the temperature in which the condensation of $\mathrm{BN}$ is possible.

Fig. 5 shows the TEM image of the samples collected in the wall of reaction cylinder V. In Fig. 5, no nanotube is observed and it is found that the catalyst particles have well crystallized structures unlike the catalyst particles shown in Fig. 4. This means that the temperature of the reaction cylinder $\mathrm{V}$ is under the meting temperature of the catalysts. From the above results, it can be concluded that the temperature in the reaction cylinder plays an important role for the formation of BNNTs in our method. The plasma conditions such as temperature, velocity, and compositions of species can 
be decided by numerical calculations and plasma diagnostic techniques. In addition, because each reaction cylinders emit optical light of different intensity during plasma operation in our experiment, the optical pyrometry methods can be used. Therefore, more intensive researches for the analysis of correlation between plasma conditions and different morphology of the samples collected in each reaction cylinders will provide an opportunity for solving the growth mechanism of BNNTs.

\section{Conclusion}

We have demonstrated the synthesis method of BNNTs by arc-jet plasma using with a non-transferred plasma torch. SEM, TEM, and EELS analyses reveal that the synthesized nanotubes have reduced number of layers and high crystallinity comparable to the nanotubes by the conventional arc discharge method. This method is a promising technique for the large-scale production of BNNTs because of its advantages of continuous operation and easy scale-up of plasma power. Our results suggest that there is a relationship between the plasma temperature and the growth of BNNTs. So, more researches are required for solving the growth mechanism of BNNTs and the optimization of plasma conditions.

\section{Acknowledgements}

This work was supported by Korea Institute of Science and Technology Evaluation and Planning (KISTEP) of the Ministry of Science and Technology in Korea. 


\section{References}

[1] N. Chopra, R. Luyken, K. Cherrey, V. Crespi, M. Cohen, S. Louie, A. Zettl, Science 269 (1995) 966.

[2] N. Chopra, A. Zettl, Solid State Commun. 105 (1998) 297.

[3] X. Blase, A. Rubio, S. Louie, M. Cohen, Europhys. Lett. 28 (1994) 335.

[4] A. Rubio, J. Corkill, M. Cohen, Phys. Rev. B 49 (1994) 5081.

[5] A. Seayad, D. Antonelli, Adv. Mater. 16 (2004) 765.

[6] I. Narita, T. Oku, Diam. Rela. Mater. 12 (2003) 1912.

[7] D. Golberg, A. Rode, Y. Bando, M. Mitome, E. Gamaly, B. Luther-Davies, Diam. Rela. Mater. 12 (2003) 1269.

[8] R. Ma, Y. Bando, T. Sato, Chem. Phys. Lett. 337 (2001) 61.

[9] D. Golberg, Y. Bando, W. Han, K. Kurashima, T. Sato, Chem. Phys. Lett. 308 (1999) 337.

[10] E. Borowiak-Palen, M. H. Rummeli, M. Knupfer, G. Behr, K. Biedermann, T. Gemming, R. J. Kalenczuk, T. Pichler, Carbon 43 (2005) 615.

[11] Y. Chen, M. Conway, J. Williams, J. Zou, J. Mater. Res. 17 (2002) 1896.

[12] S. Yong Bae, H. Won Seo, J. Park, Y. Sang Choi, J. Chul Park, S. Young Lee, Chem. Phys. Lett. 374 (2003) 534.

[13] J. Cumings, A. Zettl, Chem. Phys. Lett. 316 (2000) 211.

[14] M. Terrones, W. K. Hsu, H. Terrones, J. P. Zhang, S. Ramos, J. P. Hare, R. Castillo, K. Prassides, A. K. Cheetham, H. W. Kroto, D. R. M. Walton, Chem. Phys. Lett. 259 (1996) 568. 
[15] S. I. Choi, J. S. Nam, J. I. Kim, T. H. Hwang, J. H. Seo, S. H. Hong, to be published.

[16] S. Yoshiki, M. Yusuke, T. Hideki, K. Shojiro, Appl. Phys. Lett. 75 (1999) 929.

[17] J. Y. Huang, H. Yasuda, H. Mori, J. Am. Ceram. Soc. 83 (2000) 403. 


\section{Figure Captions}

Fig. 1. Schematic of an arc-jet plasma reactor used for BNNTs synthesis along with a non-transferred plasma torch

Fig. 2. (a) SEM image and (b) low magnification TEM image of the samples collected from the reaction cylinder II

Fig. 3. EELS analysis results showing: (a) zero-loss energy-filtered TEM image, (b) elemental map of boron, (c) elemental map of nitrogen, and (d) a representative EELS spectrum of the nanotubes

Fig. 4. TEM images showing: (a) single-walled nanotubes, (b) double-walled nanotubes, (c) multi-walled nanotube grown from a catalyst particle, and (d) multi-walled nanotubes encapsulating catalyst particles

Fig. 5. TEM image of the samples collected from the reaction cylinder $\mathrm{V}$ 


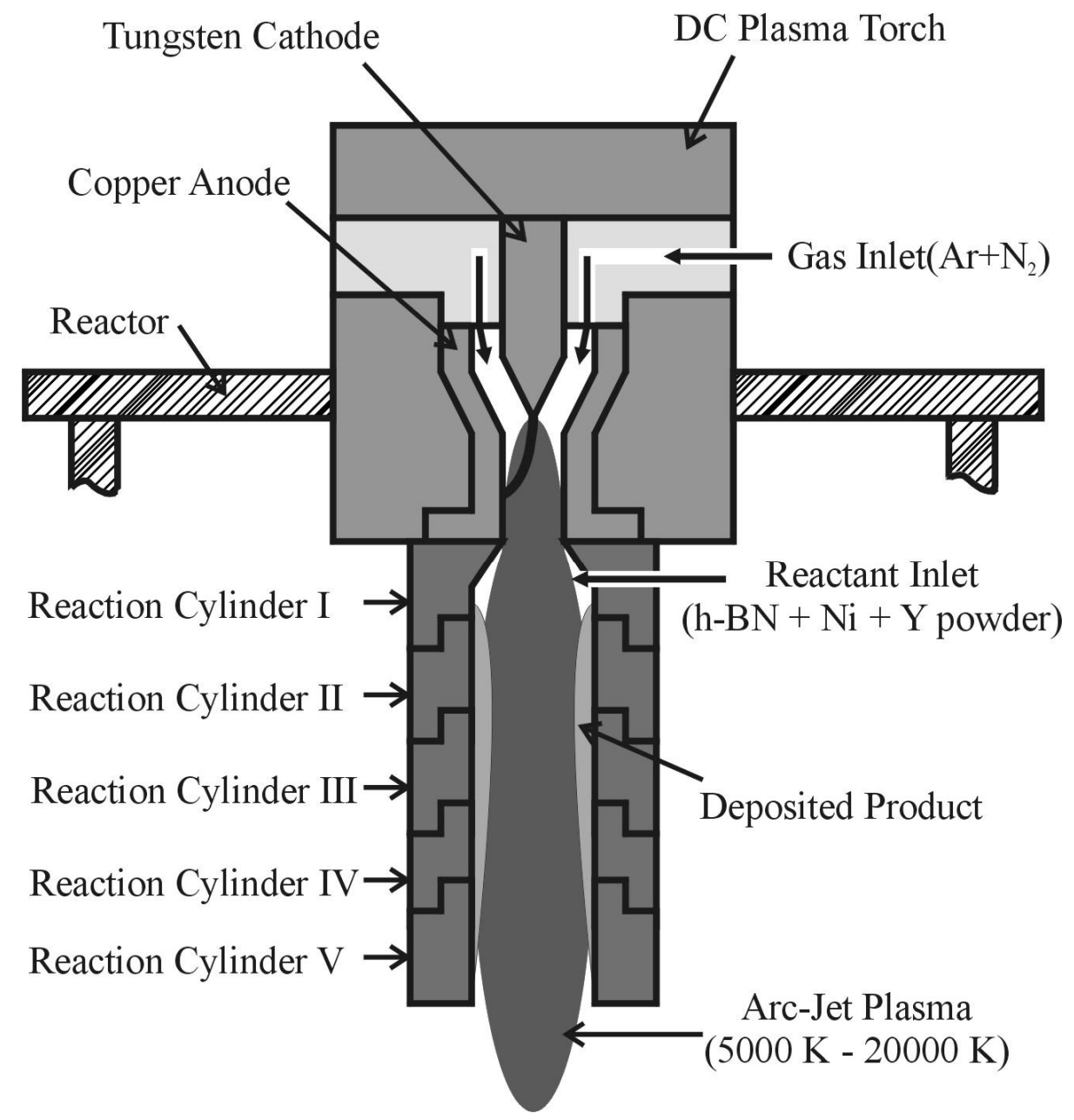

Fig. 1. 


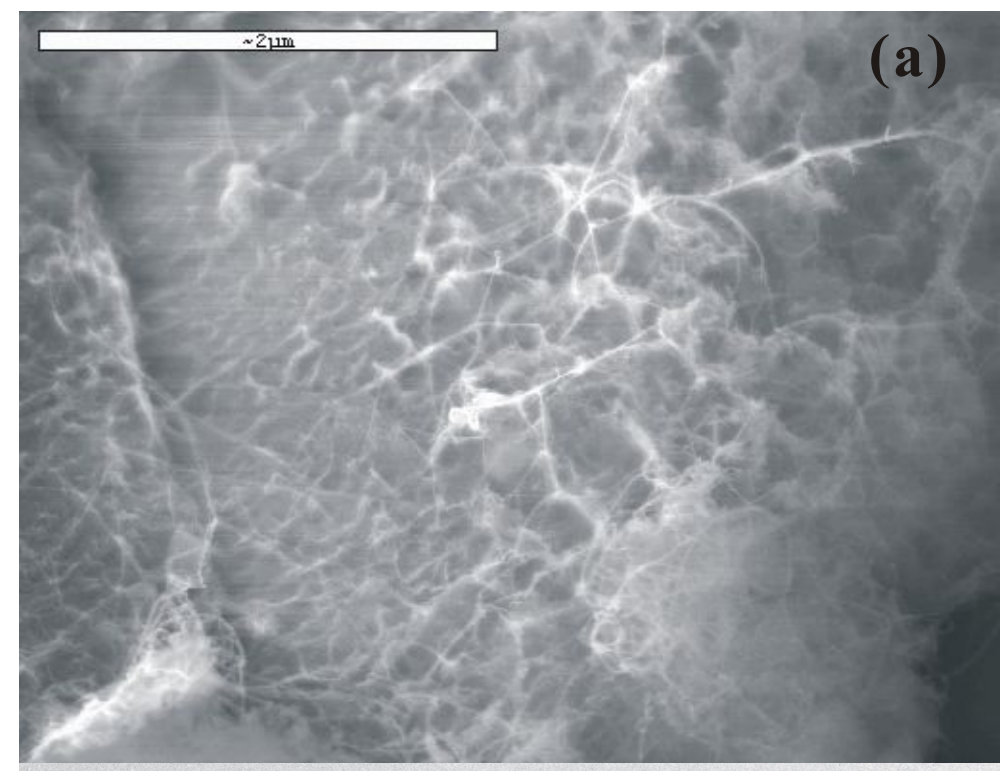

(b)

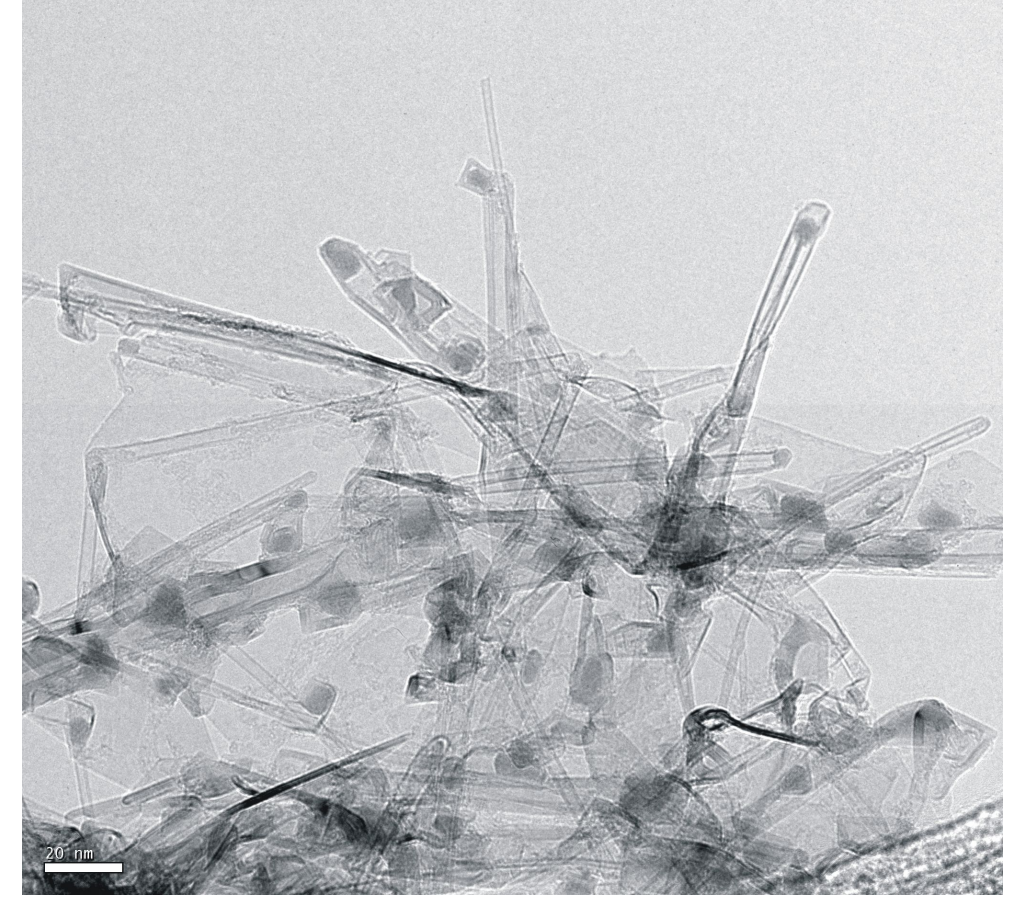

Fig. 2. 

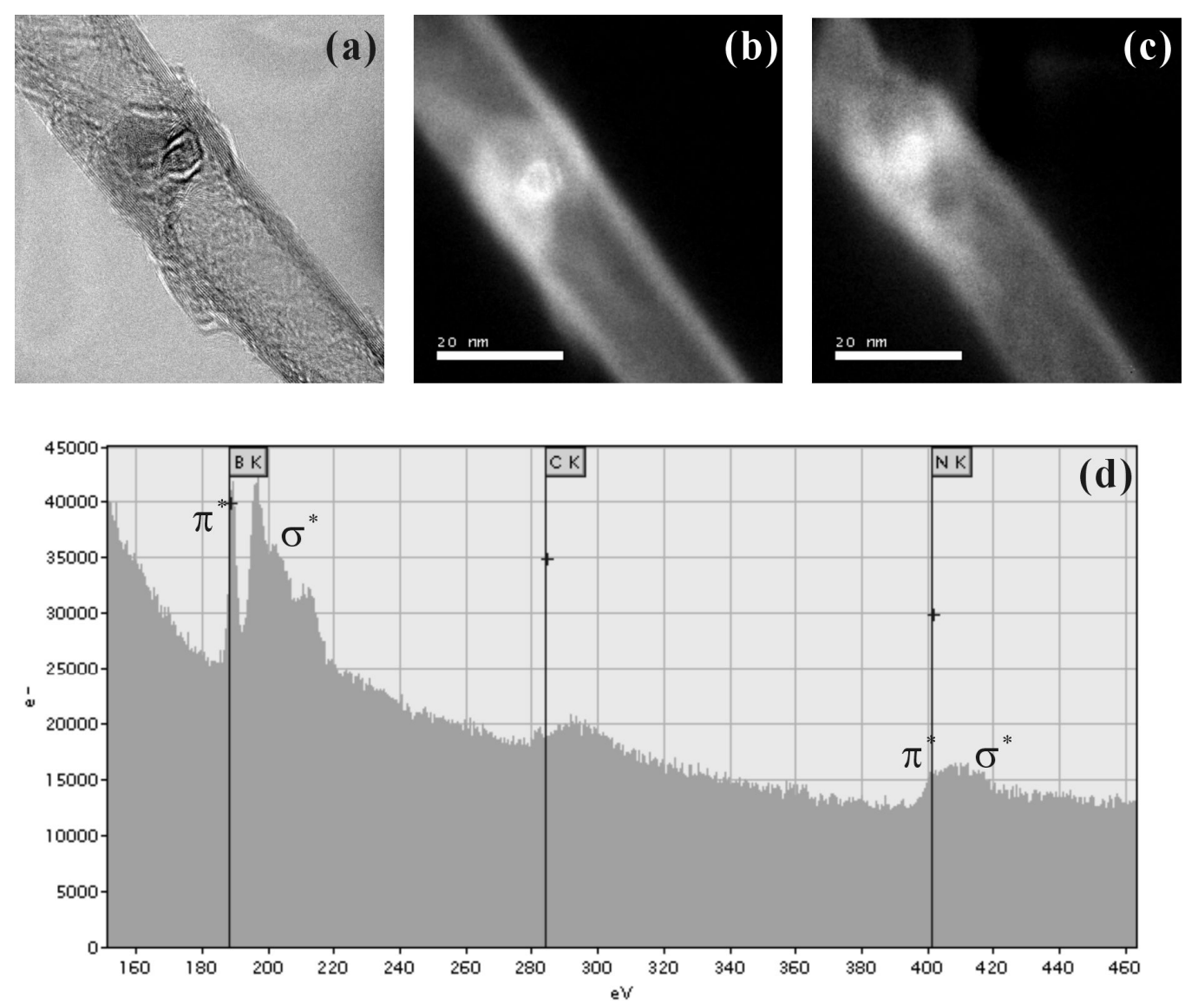

Fig. 3. 

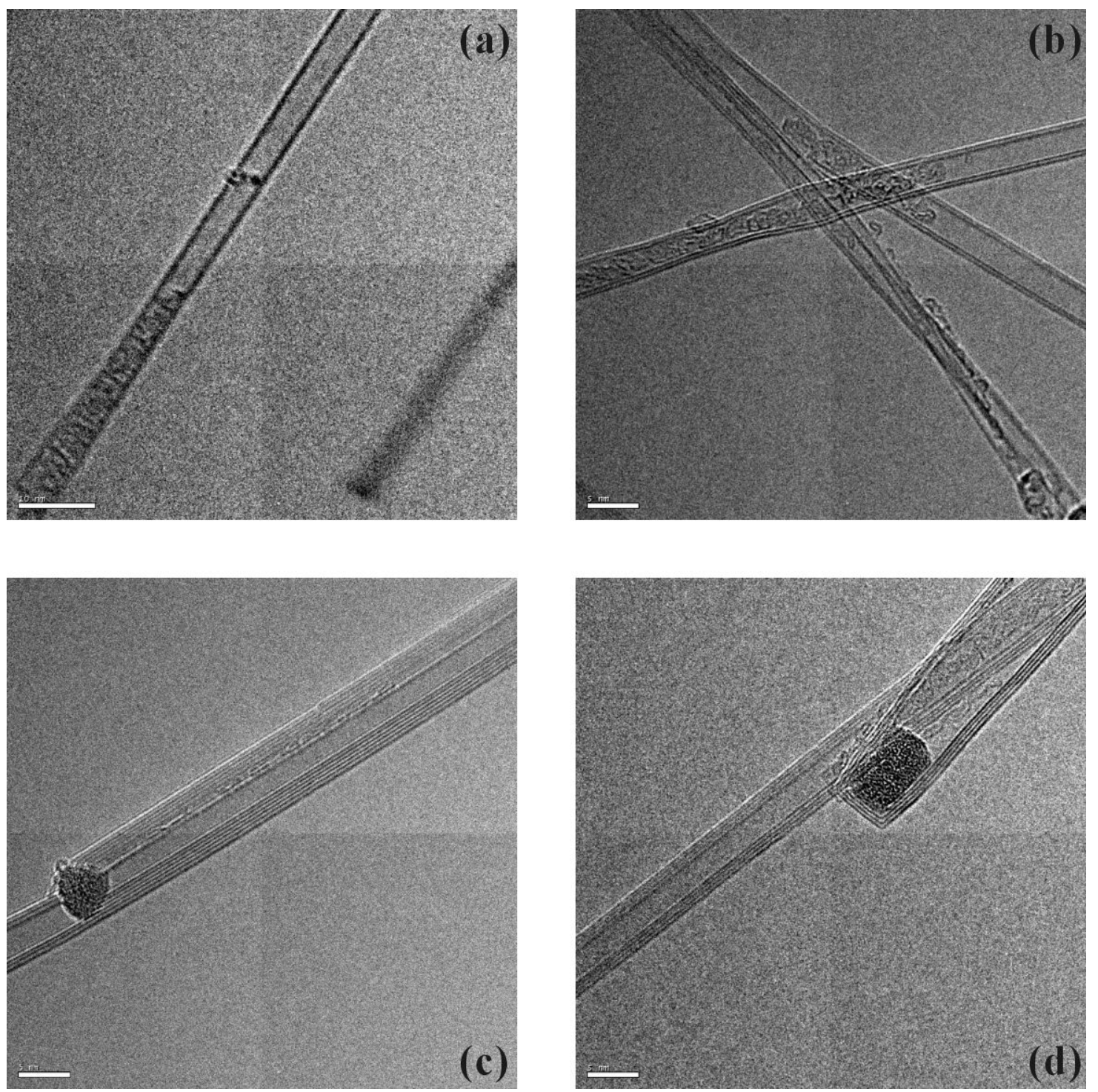

Fig. 4. 


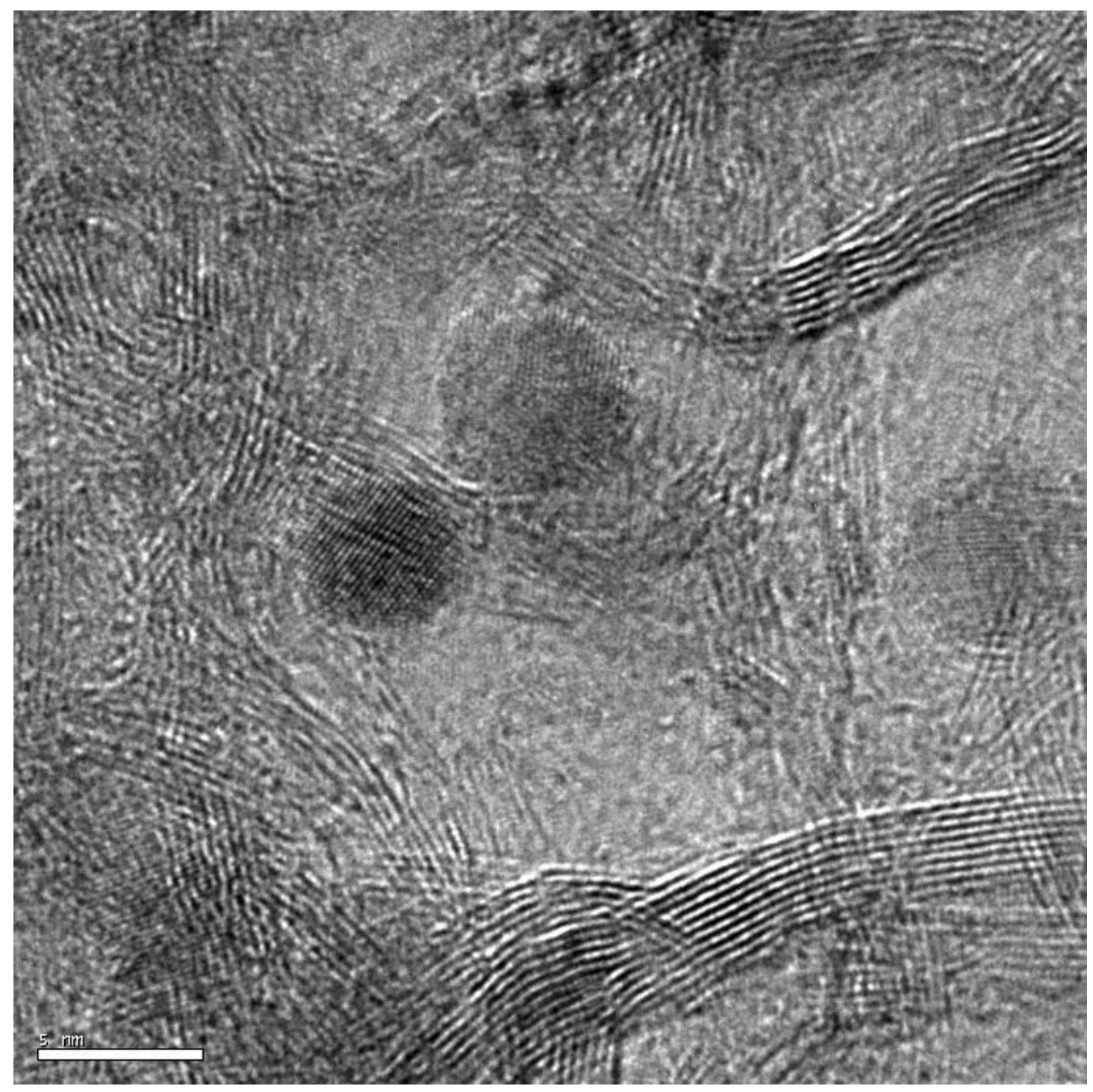

Fig. 5. 